\title{
PLANKTON DIVERSITY AND COMMUNITY STRUCTURE OF ASARAMA ESTUARY IN THE NIGER DELTA IN RELATION TO PHYSICO-CHEMISTRY
}

\author{
DiRISU, A. R. ${ }^{1 *}-$ UWAGBAE, M. A. ${ }^{1,2}-$ Edwin-Wosu, N. L. ${ }^{3}-$ IMOOBE, T. O. T. ${ }^{1}$ \\ ${ }^{I}$ Department of Animal and Environmental Biology, Faculty of Life Sciences, University of \\ Benin, Benin City, Edo State, P.M.B. 1154, Nigeria \\ ${ }^{2}$ Wetlands International, 3 a Oromineke Street, D-Line, Port Harcourt, Nigeria \\ ${ }^{3}$ Department of Plant Science and Biotechnology, University of Port Harcourt, Choba, Port \\ Harcourt, Nigeria \\ *Corresponding author \\ e-mail: dedonrahman10@yahoo.com
}

(Received 21 ${ }^{\text {st }}$ Feb 2019; accepted $15^{\text {th }}$ May 2019)

\begin{abstract}
Biodiversity of Asarama estuary at Andoni flat in the Niger Delta was surveyed in the wet and dry seasons of 2016/17; in order to ascertain the diversity, composition and community structure of plankton. Qualitative plankton samples, were collected from different sources including surface water and analysed in the laboratory. The results for the analyses for water chemistry showed optimal to near high values for the parameters, which were within the normal range of normality in tropical brackish waters. Biodiversity analyses revealed eight divisions of phytoplankton in the given order; (bacillariphyta constituting $84 \%>$ cyanophyta $8 \%>$ chlorophyta $6 \%$, > euglenophyta $1 \%$, $\geq$ dinophyta $1 \%$, > ochrophyta $<1 \%, \geq$ charophyta $<1 \%, \geq$ oligohymenophorea $<1 \%$ ) and three groups of zooplankton which were cyclopoida (composed of $98 \%$ of the species), harpaticoida and rotifera (which had $1 \%$ of the constituent species each). Species diversity and composition were low for zooplankton and very high for phytoplankton. Meanwhile, unregulated boat traffic and illegal pollution with crude oil products pose major challenges to the biotic communities. Nutrient enrichment may perhaps become an issue in the near future if no monitoring efforts backed with legislature are imposed on the maricultural practices of bivalves at the upstream.
\end{abstract}

Keywords: Andoni, inter-tidal, phytoplankton, salinity, zooplankton

\section{Introduction}

Estuaries are shallow open systems that are strongly influenced by river inflows, mixing with the coastal ocean and exchanging sediment including the cleaning of the atmosphere and the water interfaces. They usually have distinct salinity gradients in their lower part and a tidal influence in their upper freshwater part providing specific hydrological properties when compared to rivers. Importantly, estuaries form transition zones between freshwater and marine environments, and are thus characterised by a large variability in their biophysical and chemical properties under both the influence of climate change and intense anthropogenic activities (Pearle et al., 2010; Lancelot and Muylaert, 2011; Yang et al., 2014). Asarama estuary is one of the most important navigable waterways in the far reaches within Andoni land and it connects several communities therein. Besides, it is exposed to both the natural factors and human exploitation activities daily (Ansa and Francis, 2007).

The estuarine environment represents an ecotone between freshwater and marine ecosystems and is influenced by both, but is in many ways more complex than either of 
them (Cearreta et al., 2000; Elliott and De Jonge, 2002; Elliott and McLusky, 2002; Yang et al., 2014). Both river flow and tidal motions drive the riverine and marine communities towards estuaries (Waniek et al., 2005) and hence shape the diversity and abundance of estuarine communities (Elliott and McLusky, 2002; Waniek, 2003; Froneman, 2004; Cloern et al., 2014). Despite the well-documented role of zooplankton in the transfer of carbon and energy, and in ichthyofaunal abundance, relatively few studies have dealt with the determinants of zooplankton assemblages in estuarine ecosystems (Carlsson et al., 1995; Dalal and Goswami, 2001; Tan et al., 2004; Thor et al., 2005; Hwang et al., 2010).

The productivity of any water body is determined by the amount of plankton therein as they are the major primary and secondary producers. Plankton communities serve as a base for the food chain that supports the commercial fisheries (Townsend et al., 2000; Godhantaraman et al., 2003; Conde et al., 2007; Davies et al., 2009; Ogbuagu et al., 2011). They are essential tools for biomonitoring programme (Davies et al., 2009) and as such their relative high abundance and diversity is crucial.

Some available studies in the Niger Delta brackish waters dealing with phytoplankton and zooplankton include Opute (1990) on the phytoplankton of Warri/Focados estuary in Delta State; Kadiri (2006) on the survey of phytoplankton on the western Niger Delta; Davies et al. (2009) on the phytoplankton of Elechi creek, and Ogbuagu et al. (2011) on the myco-plankton of Imo River in the Niger Delta).

There is a huge dearth of knowledge on the plankton communities of Asarama estuary regarding its checklist, diversity and community structure; as no such known study was documented prior to this work upon search. Hence, this study was timely and addresses the influence of environmental activities upon the composition, diversity and community structure of the plankton of Asarama estuary at Andoni flat in the Niger Delta. Therefore, the aim and objectives of this study were to document the diversity, community composition and the physicochemical environment associated with the plankton of Asarama estuary. We used Multivariate approach such as canonical correspondence (CC) analysis to test for the associations between plankton communities and the physicochemical environment in the study area.

\section{Materials and methods}

\section{Study area}

Asarama estuary at Adoni land in Rivers State is located within the coordinates $\left(04^{\circ} 30^{\prime} 37^{\prime} \mathrm{N}, 007^{\circ} 27^{\prime} 05^{\prime \prime} \mathrm{E}\right.$ and $\left.04^{\circ} 31^{\prime} 35^{\prime \prime} \mathrm{N}, 007^{\circ} 28^{\prime} 44^{\prime \prime} \mathrm{E}\right)$. It is a semi-diurnal tidal estuary system with a bridge across to other communities near shore (Fig. 1). The sampling stations (which are designated as points 1 to 5, in yellow ink along the water course on the map) were fixed by using a hand held Global Positioning System (GPS) and were maintained throughout the study period.

\section{Sampling stations}

The ecosystem is characterised by rich flora communities of white and red mangroves, Nipa palm forests and other halophytes. At station 1, is located a forest of mangroves named; Aso mangrove forest which is over 200 years old (personal communication, 2016). The forest is a host to several species of birds, monkeys, reptiles, bees and insects as observed during field studies. Stations 2 is rich in 
mangroves as well. Decapods and bivalves were commonly seen on mangrove branches here. Stations 3 is characterised by an admixture of white mangrove and nipa palms. The sediment was mostly silty and contained a lot of dead decaying organic matter like dead woods, dead leaves and dead shells of molluscs. Station 4 is located far downstream after the bridge by the fishing wooden traps. The substratum was a mixture of sandy-muddy bed. A film of soot from an illegal petroleum local refinery was observed on the water surface throughout the study. Station 5 is equally located downstream of station 4. A lot of nipa palms were found here with so much decomposition activities of the palm fronds and their seeds. The substratum was mostly muddy at the upper most reaches.

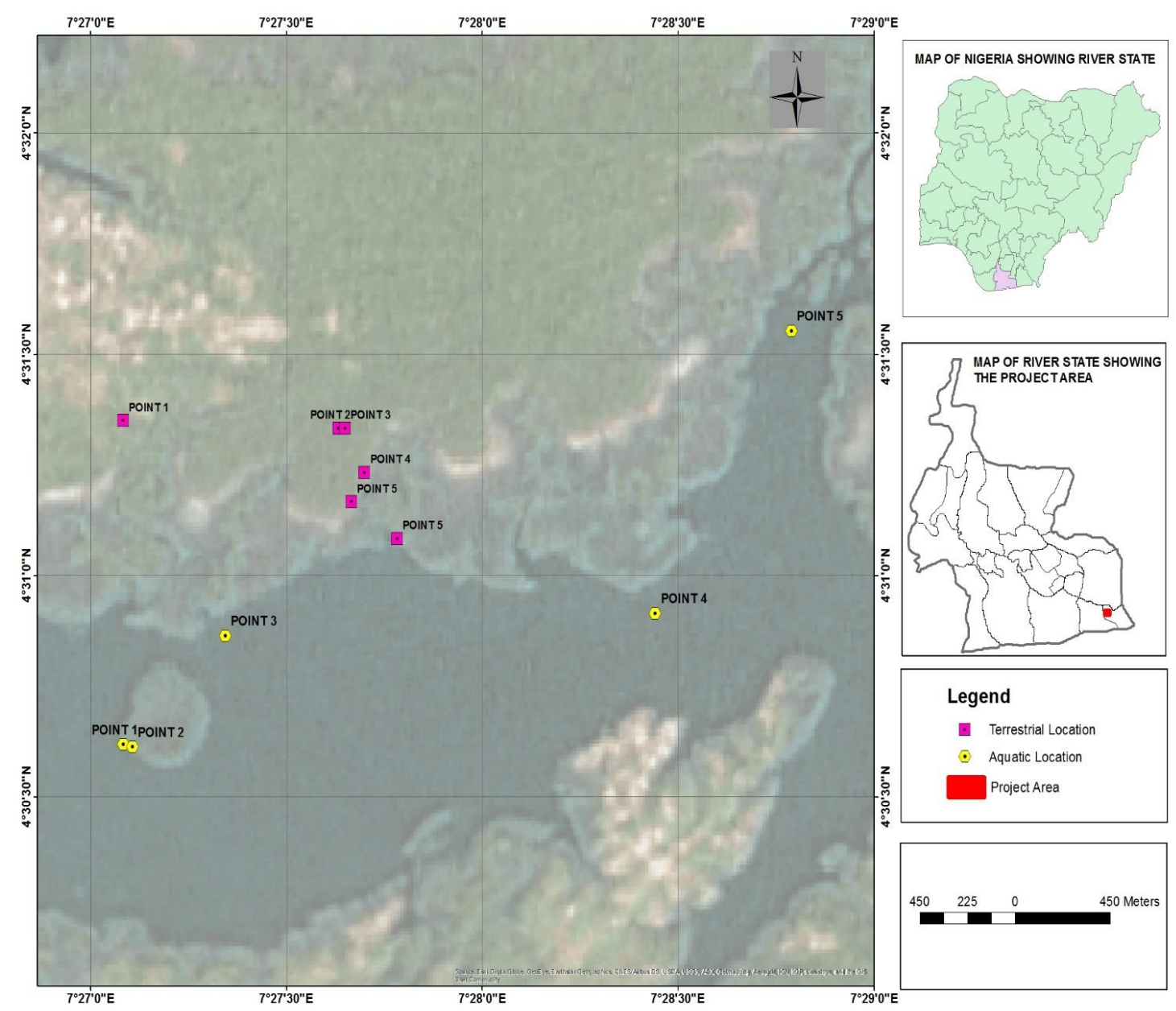

Figure 1. Map of the study area

\section{Human activities and land used pattern}

The major human activities in the locality include land and water transportations using cars, motor-bikes and boats, fishing with fish nets, gears and fish traps. Mariculture of Oysters (Castrostrea sp) and Periwinkles (Tympanotonus fuscatus Var. Radular) were predominant. At low tides particularly between 08:30 and 10:00 some of the locals were engaged in harvesting periwinkles indiscriminately without regulations. 
Illegal local refinery of petroleum products was observed far upstream off the estuary at the north-western bank which was emitting soot into the atmosphere.

\section{Surface water sampling and analyses}

Surface water samples were collected, preserved where applicable and analysed either in the field or laboratory according the methods of Eaton et al. (2005). A HANNAH field $\mathrm{pH}$ meter was used for the determination of $\mathrm{pH}$ in-situ. Turbidity was determined in the laboratory by using a HACH DR - 2000 spectrophotometer (Hach instrument). Salinity was determined in the laboratory by using HACHCO150 Model for Total Dissolved Solid/Conductivity/Salinity. This was determined using a HACHCO150 TDS/Conductivity/Salinity meter. Azide modification of Winkler's method was used to determine dissolved oxygen content of the water samples. Also, the Azide modification of Winkler's method was used to determine BOD content of the water samples. Nitrate was determined using HACH ER 2000 spectrophotometer. Determination of sulphate was done using a HACH DR 2000 spectrophotometer.

\section{Plankton sampling and laboratory analyses}

We used qualitative method to collect both phyto and zooplankton communities from the subsurface water in August 2016, the peak of wet season in the Niger Delta and March 2017 (driest month in the dry season) across five sampling stations (designated as points 1 to 5 on the map) by towing a 55 micron plankton net tied to a $25 \mathrm{HP}$ enginepowered boat cruising at a speed of about $5 \mathrm{~km} \mathrm{~h}^{-1}$ for not more than 3 minutes in all. Plankton samples were then preserved with few drops of $10 \%$ buffered formaldehyde solution in $250 \mathrm{ml}$ plastic bottles (polypropylene bottles) (Davies et al., 2009; Imoobe and Adeyinka, 2009). Samples were observed in the postgraduate laboratory of the Department of Animal and Environmental biology, University of Benin, Benin, Nigeria; using the Olympus binoculars microscope $(\mathrm{CH})$. Identification and classification to species level where possible, was carried out with the appropriate literatures (Newell and Newell, 1966; Prescott, 1975; Needham and Needham, 1978 and Van De Velde, 1984; Jeje and Fernando, 1986).

\section{Data analyses}

Physicochemical data were subjected to the Analysis of Variance (One-way ANOVA) using SPSS (version 16.0). Plankton results were tested for biological diversity indices such as species richness, Shannon diversity index and species evenness with the aid of Palaeontological Statistics software (PAST 1.99 version) and canonical correspondence analysis (CCA) was also performed to test for any existing association between environmental condition and the biota (Hammer et al., 2001; Uwagbae et al., 2017). Plankton data were equally analysed by using basic tools for the measurement of central tendency such as graphics and percentage distribution.

\section{Results}

\section{Physico-chemical environment of Asarama estuary}

The physical and chemical condition of Asarama estuary surface water is presented in Tables 1 and 2, respectively. 
Table 1. Summary of the seasonal mean and standard deviation of the physicochemical condition in surface water of Asarama estuary (between August, 2016 and March, 2017)

\begin{tabular}{c|c|c|c|c|c|c}
\hline Parameters & $\begin{array}{c}\text { Station 1 } \\
\text { Mean } \pm \text { SD }\end{array}$ & $\begin{array}{c}\text { Station 2 } \\
\text { Mean } \pm \text { SD }\end{array}$ & $\begin{array}{c}\text { Station 3 } \\
\text { Mean } \pm \text { SD }\end{array}$ & $\begin{array}{c}\text { Station 4 } \\
\text { Mean } \pm \text { SD }\end{array}$ & $\begin{array}{c}\text { Station 5 } \\
\text { Mean } \pm \text { SD }\end{array}$ & $\begin{array}{c}\text { Significant } \\
\text { values }\end{array}$ \\
\hline Air temperature $\left({ }^{\circ} \mathrm{C}\right)$ & $30.00 \pm 2.83$ & $30.00 \pm 4.24$ & $30.00 \pm 4.24$ & $30.50 \pm 3.54$ & $31.00 \pm 4.24$ & 0.998 \\
Water temperature $\left({ }^{\circ} \mathrm{C}\right)$ & $28.00 \pm 2.83$ & $27.75 \pm 1.77$ & $28.00 \pm 2.83$ & $29.00 \pm 4.24$ & $28.50 \pm 3.54$ & 0.996 \\
pH & $7.20 \pm 0.14$ & $7.30 \pm 0.14$ & $7.30 \pm 0.14$ & $7.15 \pm 0.21$ & $7.00 \pm 0.28$ & 0.554 \\
Turbidity $\left(\mathrm{NTU}^{\prime}\right)$ & $9.00 \pm 1.41$ & $19.00 \pm 8.49$ & $10.00 \pm 4.24$ & $11.00 \pm 1.41$ & $14.00 \pm 1.41$ & 0.259 \\
EC $\left(\mathrm{mSCm}^{-1}\right)$ & $8.35^{\mathrm{a}} \pm 0.28$ & $9.37^{\mathrm{a}} \pm 0.08$ & $7.82^{\mathrm{a}} \pm 0.71$ & $7.05^{\mathrm{a}} \pm 0.15$ & $5.22^{\mathrm{b}} \pm 1.27$ & 0.022 \\
TDS $\left(\mathrm{mgL}^{-1}\right)$ & $5.12^{\mathrm{a}} \pm 1.38$ & $5.53^{\mathrm{a}} \pm 5.30$ & $4.95^{\mathrm{a}} \pm 3.75$ & $4.94^{\mathrm{a}} \pm 7.01$ & $3.04^{\mathrm{b}} \pm 389$ & 0.010 \\
DO $\left(\mathrm{mgL}^{-1}\right)$ & $7.21 \pm 0.72$ & $7.32 \pm 0.28$ & $7.74 \pm 0.59$ & $7.45 \pm 0.42$ & $7.88 \pm 0.40$ & 0.669 \\
BOD $\left(\mathrm{mgL}^{-1}\right)$ & $0.98^{\mathrm{c}} \pm 0.01$ & $0.99^{\mathrm{c}} \pm 0.01$ & $1.89^{\mathrm{a}} \pm 0.16$ & $1.18^{\mathrm{bc}} \pm 0.03$ & $1.49^{\mathrm{ab}} \pm 0.38$ & 0.014 \\
Salinity $(\%)$ & $4.95 \pm 0.49$ & $5.25 \pm 0.07$ & $4.55 \pm 0.78$ & $4.25 \pm 0.64$ & $3.20 \pm 1.27$ \\
Total hardness $\left(\mathrm{CaCO}_{3}\right)\left(\mathrm{mgL}^{-1}\right)$ & $1160.00 \pm 70.71$ & $1430.00 \pm 14.14$ & $1075.00 \pm 91.92$ & $1075.00 \pm 162.63$ & $1210.00 \pm 424.26$ & 0.234 \\
Nitrate $\left(\mathrm{mgL}^{-1}\right)$ & $0.13 \pm 0.03$ & $0.10 \pm 0.01$ & $0.14 \pm 0.03$ & $0.12 \pm 0.04$ & $0.09 \pm 0.01$ & 0.378 \\
Phosphate $\left(\mathrm{mgL}^{-1}\right)$ & $0.30^{\mathrm{c}} \pm 0.03$ & $0.34^{\mathrm{bc}} \pm 0.04$ & $0.42^{\mathrm{ab}} \pm 0.04$ & $0.44^{\mathrm{ab}} \pm 0.04$ & $0.46^{\mathrm{a}} \pm 0.04$ & 0.033 \\
Sulphate $\left(\mathrm{mgL}^{-1}\right)$ & $358.50 \pm 44.55$ & $455.00 \pm 91.92$ & $350.00 \pm 42.43$ & $311.00 \pm 26.87$ & $254.00 \pm 79.20$ & 0.157 \\
\hline
\end{tabular}

Significant < 0.05 : significant difference. Superscript denotes the source of significant variation

Table 2. Mean values of the investigated parameters in surface water across wet and dry seasonal during the study

\begin{tabular}{c|c|c|c}
\hline Parameters & $\begin{array}{c}\text { Wet season } \\
\text { Mean } \pm \text { SD }\end{array}$ & $\begin{array}{c}\text { Dry season } \\
\text { Mean } \pm \text { SD }\end{array}$ & Significant \\
\hline Air temperature $\left({ }^{\circ} \mathrm{C}\right)$ & $27.60 \pm 0.55$ & $33.00 \pm 0.71$ & 0.000 \\
Water temperature $\left({ }^{\circ} \mathrm{C}\right)$ & $26.10 \pm 0.22$ & $30.40 \pm 1.14$ & 0.000 \\
$\mathrm{pH}$ & $7.06 \pm 0.17$ & $7.32 \pm 0.08$ & 0.015 \\
Turbidity $(\mathrm{NTU})$ & $15.00 \pm 5.87$ & $10.20 \pm 2.77$ & 0.107 \\
EC $\left(\mathrm{mSCm}^{-1}\right)$ & $7.21 \pm 1.85$ & $7.91 \pm 1.29$ & 0.480 \\
TDS $\left(\mathrm{mgL}^{-1}\right)$ & $4.61 \pm 1.19$ & $4.84 \pm 0.86$ & 0.700 \\
DO $\left(\mathrm{mgL}^{-1}\right)$ & $7.86 \pm 0.29$ & $7.18 \pm 0.33$ & 0.008 \\
BOD $\left(\mathrm{mgL}^{-1}\right)$ & $1.38 \pm 0.47$ & $1.23 \pm 0.33$ & 0.599 \\
Salinity $(\%)$ & $3.98 \pm 1.09$ & $4.90 \pm 0.51$ & 0.139 \\
Total hardness $\left(\mathrm{CaCO}_{3}\right)\left(\mathrm{mgL}^{-1}\right)$ & $1108.00 \pm 199.67$ & $1272.00 \pm 202.53$ & 0.223 \\
Nitrate $\left(\mathrm{mgL}^{-1}\right)$ & $0.13 \pm 0.03$ & $0.10 \pm 0.02$ & 0.038 \\
Phosphate $\left(\mathrm{mgL}^{-1}\right)$ & $0.42 \pm 0.07$ & $0.36 \pm 0.07$ & 0.252 \\
Sulphate $\left(\mathrm{mgL}^{-1}\right)$ & $386.00 \pm 82.04$ & $305.40 \pm 69.91$ & 0.132 \\
\hline
\end{tabular}

Significant $<0.05$ : significant difference

Air and water temperatures closely followed each other. The differences between air and water temperatures was not more than $4{ }^{\circ} \mathrm{C}$. Mean air temperature ranged between 30 and $31^{\circ} \mathrm{C}$ across the stations. Likewise water temperature varied from $27.75^{\circ} \mathrm{C}$ at station 2 to $29{ }^{\circ} \mathrm{C}$ at station 4 . There was however no significant differences between the two parameters $(\mathrm{P}>0.05)$.

The mean spatial and temporal concentrations of hydrogen ion concentration $(\mathrm{pH})$ values showed an alkaline condition ranging between 7.00 at station 5 and 7.30 at stations 2 and 3 respectively. The mean values across stations 1 to 5 in wet and dry seasons were between 7.06 and 7.32. Turbidity mean wet and dry season values ranged from 10.20 to $15.00 \mathrm{NTU}$. Turbidity mean concentration values were between $900 \mathrm{NTU}$ at station 1 and $19.00 \mathrm{NTU}$ at station 2. Electrical conductivity (EC) mean seasonal concentrations were between $5.22 \mathrm{mScm}^{-1}$ at station 5 and $9.37 \mathrm{mScm}^{-1}$ at station 2 . 
Total hardness and nutrients (phosphate, sulphate and nitrate) exhibited condition tenable in the marine environment. Mean values of total hardness varied from $1075.00 \mathrm{mgL}^{-1}$ at station 3 and 4 respectively to $1430.00 \mathrm{mgL}^{-1}$ at station 2 . Its mean concentration value in the water was between $1108.00 \mathrm{mgL}^{-1}$ in the wet season and $1272.00 \mathrm{mgL}^{-1}$ in the dry season. Nitrate and phosphates both had low range of values. The mean temporal and spatial concentration values were less than $0.5 \mathrm{mgL}^{-1}$. However, sulphate recorded very high values of between $386.00 \mathrm{mgL}^{-1}$ during the wet season and $305.40 \mathrm{mgL}^{-1}$ during the dry season. Sulphate mean concentration were between $254.00 \mathrm{mgL}^{-1}$ at station 5 and $455.00 \mathrm{mgL}^{-1}$ at station 2 . The seasonal values of dissolved oxygen (DO) ranged between $7.18 \mathrm{mgL}^{-1}$ in the dry season and $7.86 \mathrm{mgL}^{-1}$ in the wet season. Mean values across the stations ranged from $7.21 \mathrm{mgL}^{-1}$ at station 1 to $788 \mathrm{mgL}^{-1}$ at station 5. Biological oxygen demand (BOD 5 ) seasonal values ranged from $1.23 \mathrm{mgL}^{-1}$ in the dry season to $1.38 \mathrm{mgL}^{-1}$ in the wet season. And mean values showed variations from $0.98 \mathrm{mgL}^{-1}$ at station 1 to $1.89 \mathrm{mgL}^{-1}$ at station 3 .

Salinity mean concentrations varied from $3.20 \mathrm{ppm}$ at station 5 to $4.95 \mathrm{ppm}$ at station 1. Seasonal concentrations varied between $3.98 \mathrm{ppm}$ in wet season and $4.90 \mathrm{ppm}$ in the dry season.

\section{Plankton studies}

Both phyto- and zooplankton were characterised and presented in this section.

\section{Phytoplankton}

Phytoplankton analysis revealed a total of eight divisions dominated by the Bacillariphyta constituting about 84\%. Others were in the order Cyanophyta $(8 \%)>$ Chlorophyta $(6 \%),>$ Eglenophyta $\quad(1 \%), \geq$ Dinophyta $\quad(1 \%),>$ Ochrophyta $(<1 \%), \geq$ Charophyta $(<1 \%), \geq$ Oligohymenophorea $(<1 \%)$ (Figs. 2 and 3$)$. The total number of individuals is represented by 9,276 individuals per meter squared, amongst 57 taxa. Phytoplankton taxa had higher percentage composition and distribution across the study stations except at station 5 and were significantly higher during the dry season of the study in 2017 (Table 3).

\section{Percentage Composition}

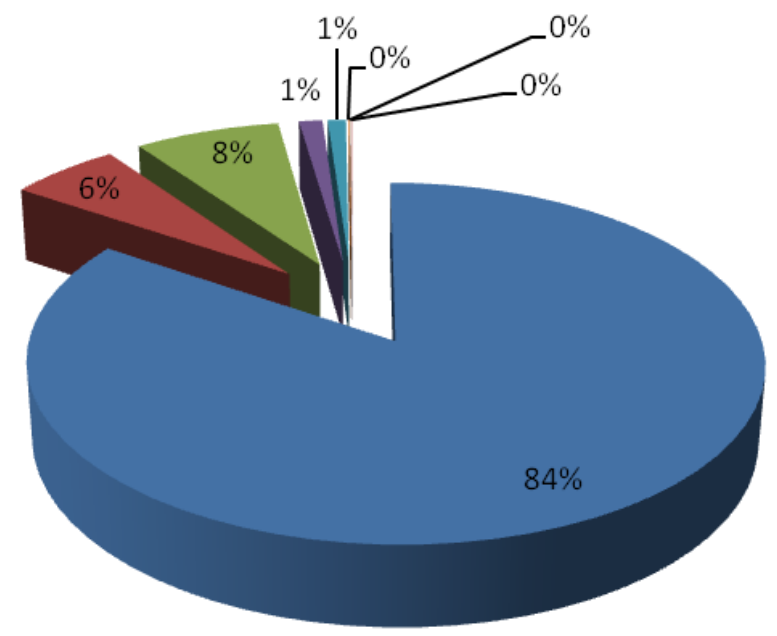

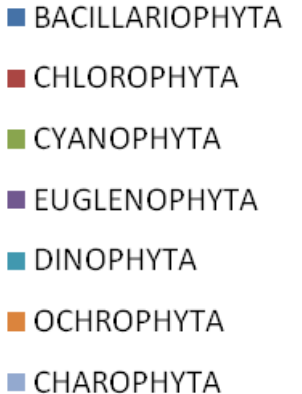

- OLIGOHYMENOPHOREA

Figure 2. Percentage composition of phytoplankton divisions 


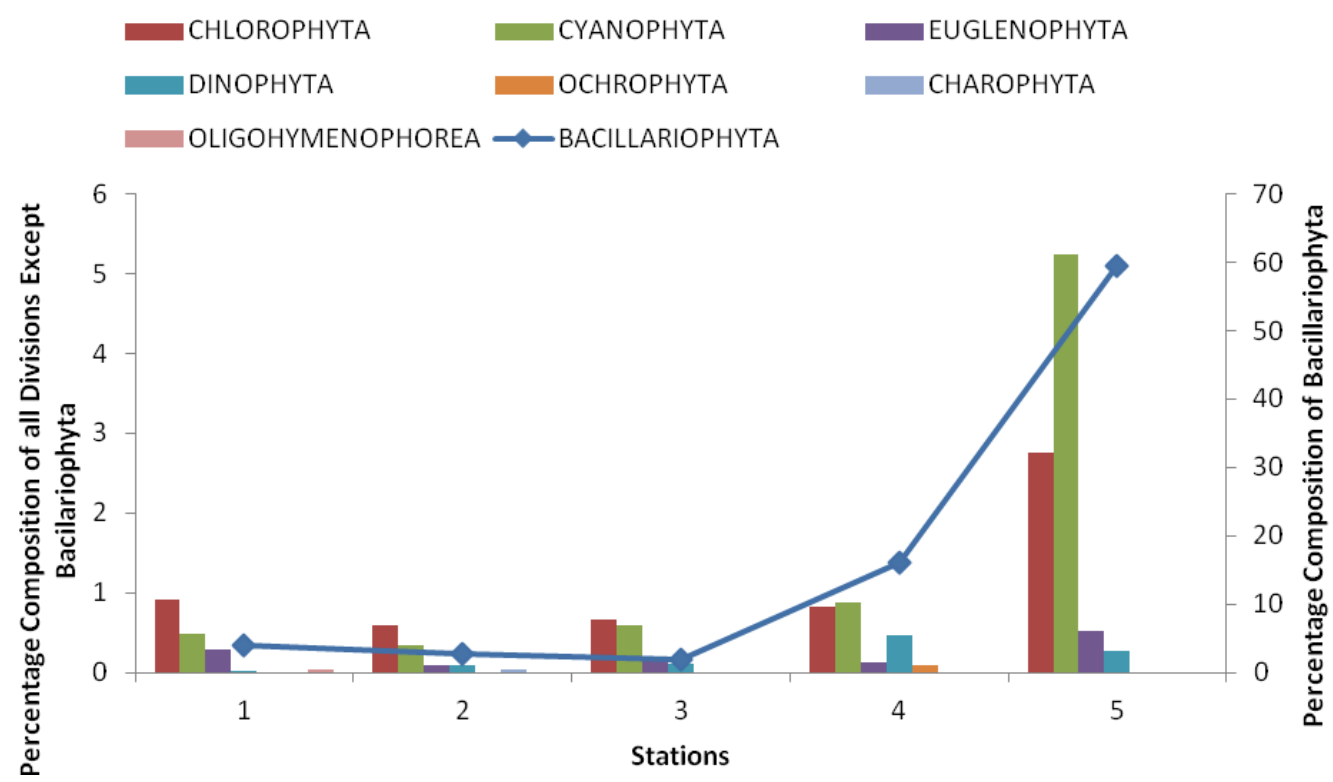

Figure 3. Percentage composition and distribution of phytoplankton groups across the study stations

Table 3. Summary of the composition and distribution of phytoplankton species across the study stations

\begin{tabular}{c|c|c|c|c|c|c}
\hline \multirow{2}{*}{ Phytoplankton species } & \multicolumn{7}{|c}{ Stations } \\
\cline { 2 - 6 } & $\mathbf{1}$ & $\mathbf{2}$ & $\mathbf{3}$ & $\mathbf{4}$ & $\mathbf{5}$ & Total \\
\hline DIVISION: BACILLARIOPHYTA & & & & & & \\
Actinoptychus splendens & 0 & 2 & 0 & 0 & 0 & 2 \\
Amphiprora alata & 0 & 3 & 0 & 0 & 0 & 3 \\
Bacillaria paradoxa & 10 & 18 & 32 & 244 & 1446 & 1750 \\
Bacillaria sp. & 0 & 0 & 0 & 0 & 48 & 48 \\
Biidulphia regia & 0 & 6 & 0 & 0 & 0 & 6 \\
Chaetoreros sp. & 4 & 0 & 0 & 0 & 0 & 4 \\
Coscinodiscus cencinnus & 1 & 0 & 0 & 0 & 0 & 1 \\
Eunotia flexuosa & 4 & 0 & 0 & 0 & 0 & 4 \\
Flagillaria javanica & 0 & 0 & 0 & 0 & 1200 & 1200 \\
Flagillaria sp. & 79 & 31 & 96 & 1216 & 2664 & 4086 \\
Melosira granulata & 4 & 0 & 0 & 0 & 0 & 4 \\
Pinnularia cardinaliculus & 4 & 0 & 0 & 0 & 0 & 4 \\
Pinnularia sp. & 0 & 0 & 6 & 12 & 24 & 42 \\
Surirella robusta & 1 & 0 & 0 & 0 & 0 & 1 \\
Surirella sp. & 3 & 0 & 0 & 0 & 60 & 63 \\
Synedra acus & 152 & 76 & 24 & 0 & 0 & 252 \\
S. ulna & 112 & 80 & 20 & 0 & 0 & 212 \\
Tabellaria floculosa & 0 & 40 & 0 & 0 & 0 & 40 \\
\hline TOTAL BACILLARIOPHYTA & 374 & 256 & 178 & 1472 & 5442 & 7722 \\
\hline DIVISION: CHLOROPHYTA & & & & & & \\
Clostrerium gracile & 4 & 4 & 0 & 0 & 0 & 8 \\
Cosmarium bretum & 0 & 0 & 0 & 0 & 48 & 48 \\
Cosmarium connatum & 4 & 0 & 0 & 0 & 0 & 4
\end{tabular}




\begin{tabular}{|c|c|c|c|c|c|c|}
\hline Cosmarium pseudoconnatum & 4 & 0 & 0 & 0 & 0 & 4 \\
\hline Cosmarium resiforme & 0 & 0 & 4 & 0 & 0 & 4 \\
\hline Eudorina elegans & 0 & 11 & 0 & 0 & 0 & 11 \\
\hline Mougeotia sp. & 4 & 0 & 0 & 0 & 0 & 4 \\
\hline Pandorina morum & 18 & 0 & 0 & 0 & 0 & 18 \\
\hline Pandorina sp. & 18 & 8 & 40 & 0 & 0 & 66 \\
\hline Pediastrum gracillinum & 0 & 0 & 0 & 0 & 72 & 72 \\
\hline Pleodorina illinosensis & 0 & 1 & 0 & 0 & 0 & 1 \\
\hline Scenedesemus dimorphus & 0 & 0 & 0 & 8 & 0 & 8 \\
\hline Scenedesemus opollensis & 0 & 0 & 0 & 8 & 0 & 8 \\
\hline Sirogonium melanosporum & 26 & 8 & 8 & 8 & 48 & 98 \\
\hline Spirogyra karnalae & 0 & 0 & 0 & 0 & 24 & 24 \\
\hline Spirogyra sp. & 4 & 0 & 0 & 0 & 0 & 4 \\
\hline Volvox africana & 2 & 10 & 0 & 32 & 36 & 80 \\
\hline Volvox aureus & 0 & 12 & 8 & 20 & 24 & 64 \\
\hline TOTAL CHLOROPHYTA & 84 & 54 & 60 & 76 & 252 & 526 \\
\hline \multicolumn{7}{|l|}{ DIVISION: CYANOPHYTA } \\
\hline Aphanothecae sp. & 0 & 0 & 16 & 0 & 0 & 16 \\
\hline Coelosphaerium sp. & 24 & 8 & 32 & 32 & 168 & 264 \\
\hline Coelosphaerium pallidum & 0 & 0 & 0 & 48 & 288 & 336 \\
\hline Microcystis aeruginosa & 3 & 0 & 0 & 0 & 0 & 3 \\
\hline Microcystis flos-aquae & 0 & 0 & 0 & 0 & 24 & 24 \\
\hline Oscillatoria bornettia & 11 & 0 & 0 & 0 & 0 & 11 \\
\hline Oscillatoria sp. & 0 & 24 & 0 & 0 & 0 & 24 \\
\hline Plectonema sp. & 6 & 0 & 0 & 0 & 0 & 6 \\
\hline Lyngba aestuarri & 0 & 0 & 6 & 0 & 0 & 6 \\
\hline TOTAL CYANOPHYTA & 44 & 32 & 54 & 80 & 480 & 690 \\
\hline \multicolumn{7}{|l|}{ DIVISION: EUGLENOPHYTA } \\
\hline Euglena sp. & 18 & 0 & 0 & 8 & 48 & 74 \\
\hline Euglena spirogyra & 0 & 8 & 16 & 0 & 0 & 24 \\
\hline Euglena viridia & 5 & 0 & 0 & 0 & 0 & 5 \\
\hline Phacus tontus & 1 & 0 & 0 & 0 & 0 & 1 \\
\hline Trachelomonas eurysloma & 3 & 0 & 0 & 0 & 0 & 3 \\
\hline Trachelomonas oblonga & 0 & 0 & 0 & 4 & 0 & 4 \\
\hline TOTAL EUGLENOPHYTA & 27 & 8 & 16 & 12 & 48 & 111 \\
\hline \multicolumn{7}{|l|}{ DINOPHYTA } \\
\hline Gymnodinium fuscum & 1 & 9 & 10 & 42 & 24 & 86 \\
\hline \multicolumn{7}{|l|}{ TOTAL DINOPHYTA } \\
\hline OCHROPHYTA & & & & & & \\
\hline Gonyostomum semen & 0 & 0 & 0 & 8 & 0 & 8 \\
\hline TOTAL OCHROPHYTA & 0 & 0 & 0 & 8 & 0 & 8 \\
\hline \multicolumn{7}{|l|}{ CHAROPHYTA } \\
\hline Actinotaenium cucurbitinum & 12 & 0 & 28 & 32 & 0 & 72 \\
\hline Euastrum elegans & 0 & 0 & 0 & 24 & 24 & 48 \\
\hline Nitella gracilis & 6 & 0 & 0 & 0 & 0 & 6 \\
\hline Straurodesmus leptodermus & 0 & 3 & 0 & 0 & 0 & 3 \\
\hline TOTAL CHAROPHYTA & 0 & 3 & 0 & 0 & 0 & 3 \\
\hline OLIGOHYMENOPHOREA & & & & & & \\
\hline Epistylis plicatilis & 4 & 0 & 0 & 0 & 0 & 4 \\
\hline TOTAL OLIGOHYMENOPHOREA & 4 & 0 & 0 & 0 & 0 & 4 \\
\hline
\end{tabular}




\section{Zooplankton}

The zooplankton taxa were poorly represented and distributed in three groups; Cyclopoida, Harpaticoida and Rotifera of the phylum Arthropoda and sub-phylum Crustacea. A total of 1,299 individuals per unit area represented in 15 taxa were recorded in this aspect of the study. The group Cyclopoida constituted $98 \%$ of species while Harpaticoida and Rotifera had $1 \%$ each. Taxa composition and distribution varied irregularly across the sampled stations (Figs. 4 and 5). Meanwhile, Rotifera was highest at station 5. There was no zooplankton recorded at station 4 throughout the study (Table 4).

\section{Percentage Composition}

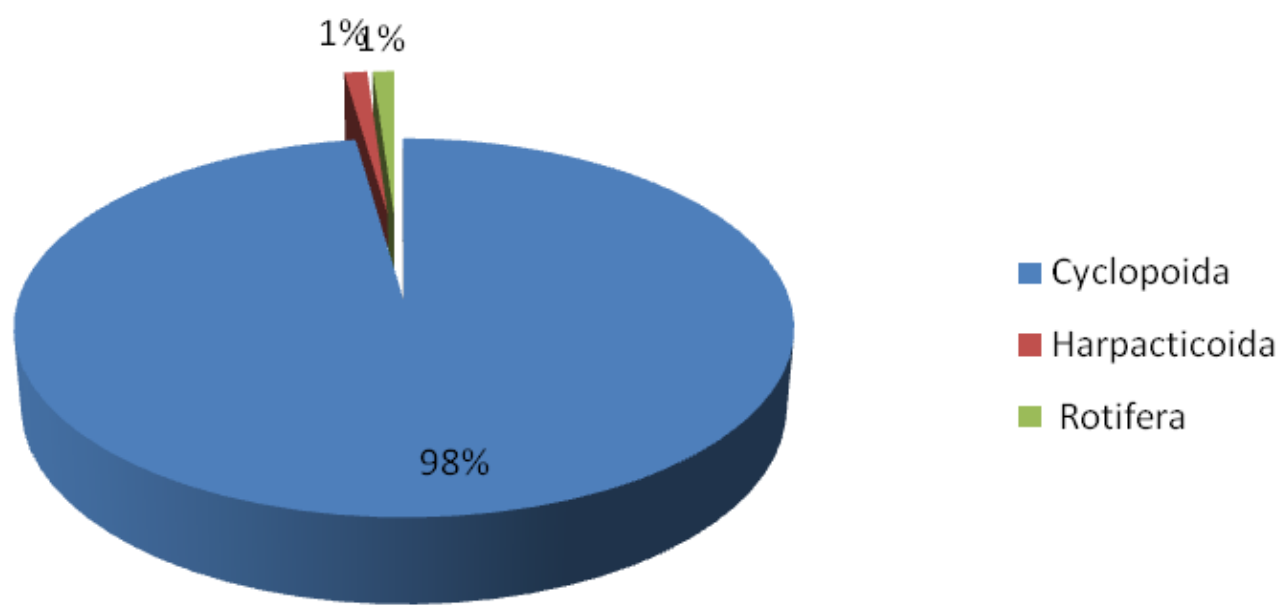

Figure 4. Percentage composition of zooplankton taxa in study area

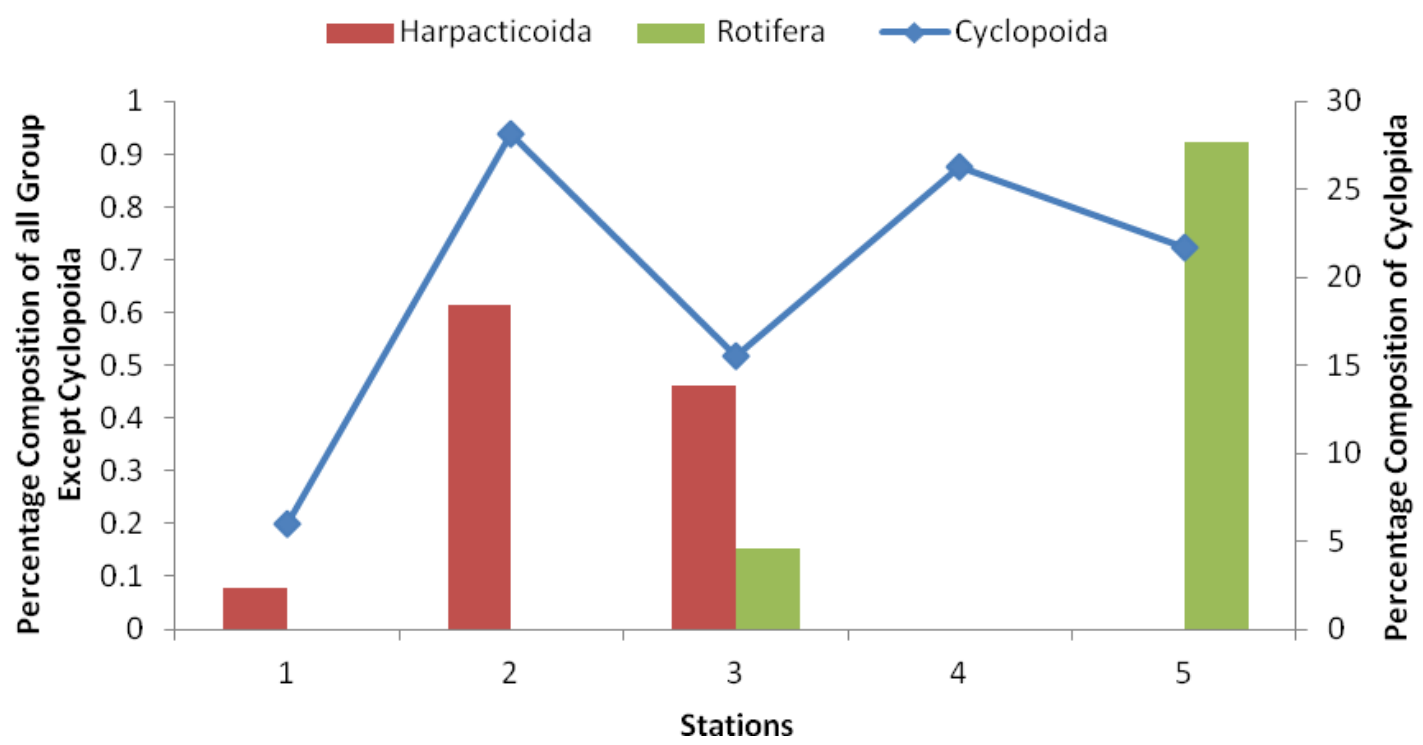

Figure 5. Percentage composition and distribution of zooplankton species across the sampled stations 
Table 4. Summary of the composition and distribution of zooplankton taxa across the study stations

\begin{tabular}{c|c|c|c|c|c|c}
\hline \multirow{2}{*}{ Zooplankton species } & \multicolumn{5}{|c}{ Stations } \\
\cline { 2 - 7 } & $\mathbf{1}$ & $\mathbf{2}$ & $\mathbf{3}$ & $\mathbf{4}$ & $\mathbf{5}$ & Total \\
\hline Phylum: Arthropoda & & & & & & \\
Subphylum: Crustacea & & & & & & \\
Subclass: Copepoda & & & & & & \\
Cyclopoida & & & & & & \\
Thermocyclops neglectus & 26 & 46 & 100 & 38 & 90 & 300 \\
Mesocyclops bodanicola & 32 & 256 & 84 & 272 & 120 & 764 \\
Metacyclops minutus & 4 & 0 & 0 & 0 & 0 & 4 \\
Microcyclops javanus & 0 & 0 & 2 & 0 & 24 & 26 \\
Acanthocyclops sp. & 6 & 48 & 8 & 0 & 48 & 110 \\
Eucyclops macruroides & 10 & 0 & 8 & 32 & 0 & 50 \\
Achidius (Neotachidius) triangular & 0 & 16 & 0 & 0 & 0 & 16 \\
Harpacticoida & & & & & & \\
Shizopera neglecta & 1 & 8 & 6 & 0 & 0 & 15 \\
\hline TOTAL COPEPODA & 79 & 374 & 208 & 342 & 282 & 1285 \\
\hline Rotifera & & & & & & \\
Keratella hiemalis & 0 & 0 & 0 & 0 & 12 & 12 \\
Rotaria neptunia & 0 & 0 & 2 & 0 & 0 & 2 \\
\hline TOTAL ROTIFERA & 0 & 0 & 2 & 0 & 12 & 14 \\
\hline
\end{tabular}

\section{Diversity indices for plankton}

Biological diversity index for phytoplankton with respect to species richness was highest at station 1 and least at station 2 . Species were only evenly distributed at stations 2 and 3. Meanwhile species dominance was highest at station 4 and Shannon diversity was least at stations 4 and 5 respectively (Table 5).

Table 5. Phytoplankton diversity index

\begin{tabular}{c|c|c|c|c|c|c}
\hline Description & STN 1 & STN 2 & STN 3 & STN 4 & STN 5 & Total \\
\hline Taxa_S & 32 & 20 & 15 & 16 & 18 & $\mathbf{1 0 1}$ \\
Individuals & 552 & 362 & 346 & 1746 & 6270 & $\mathbf{9 2 7 6}$ \\
Dominance_D & 0.1469 & 0.1253 & 0.1291 & 0.5074 & 0.2737 & \\
Shannon_H & 2.455 & 2.434 & 2.352 & 1.217 & 1.659 & \\
Simpson_1-D & 0.8531 & 0.8747 & 0.8709 & 0.4926 & 0.7263 & \\
Evenness_e^H/S & 0.364 & 0.57 & 0.7004 & 0.2111 & 0.292 & \\
Menhinick & 1.362 & 1.051 & 0.8064 & 0.3829 & 0.2273 & \\
Margalef & 4.91 & 3.225 & 2.395 & 2.009 & 1.944 & \\
Equitability_J & 0.7084 & 0.8123 & 0.8685 & 0.4389 & 0.5741 & \\
Fisher_alpha & 7.398 & 4.559 & 3.196 & 2.433 & 2.272 & \\
Berger-Parker & 0.2754 & 0.221 & 0.2775 & 0.6964 & 0.4249 & \\
\hline
\end{tabular}


For the zooplankton, species richness was observed at stations 1 and 3 only, and evenness was least at stations 3. Dominance of species occurred at station 4 and fairly at station 4 (Table 6).

Table 6. Zooplankton diversity index

\begin{tabular}{c|c|c|c|c|c|c}
\hline Description & STN 1 & STN2 & STN 3 & STN 4 & STN 5 & Total \\
\hline Taxa_S & 6 & 5 & 7 & 3 & 5 & $\mathbf{2 6}$ \\
Individuals & 79 & 374 & 210 & 342 & 294 & $\mathbf{1 2 9 9}$ \\
Dominance_D & 0.2969 & 0.5024 & 0.3907 & 0.6536 & 0.2953 & \\
Shannon_H & 1.396 & 0.9978 & 1.159 & 0.6479 & 1.359 & \\
Simpson_1-D & 0.7031 & 0.4976 & 0.6093 & 0.3464 & 0.7047 & \\
Evenness_e^H/S & 0.6729 & 0.5425 & 0.4553 & 0.6372 & 0.7786 & \\
Margalef & 1.144 & 0.6752 & 1.122 & 0.3428 & 0.7038 & \\
Equitability_J & 0.7789 & 0.62 & 0.5956 & 0.5898 & 0.8445 & \\
\hline
\end{tabular}

\section{Application of canonical correspondence analysis (CCA) on the biota}

We used Canonical correspondence analyses to characterise the relationships between the abiotic and biotic components of Asarama estuary.

For phytoplankton several associations were found at axis 2 of the CCA (Fig. 6). There was a positive association between phosphorus, sulphur and Ochrophyta species at plot 4. At plot 1, there was a positive association between $\mathrm{pH}$ and Charophyta. Meanwhile, Cyanophyta and Euglenophyta showed some positive correlations with nitrate respectively.

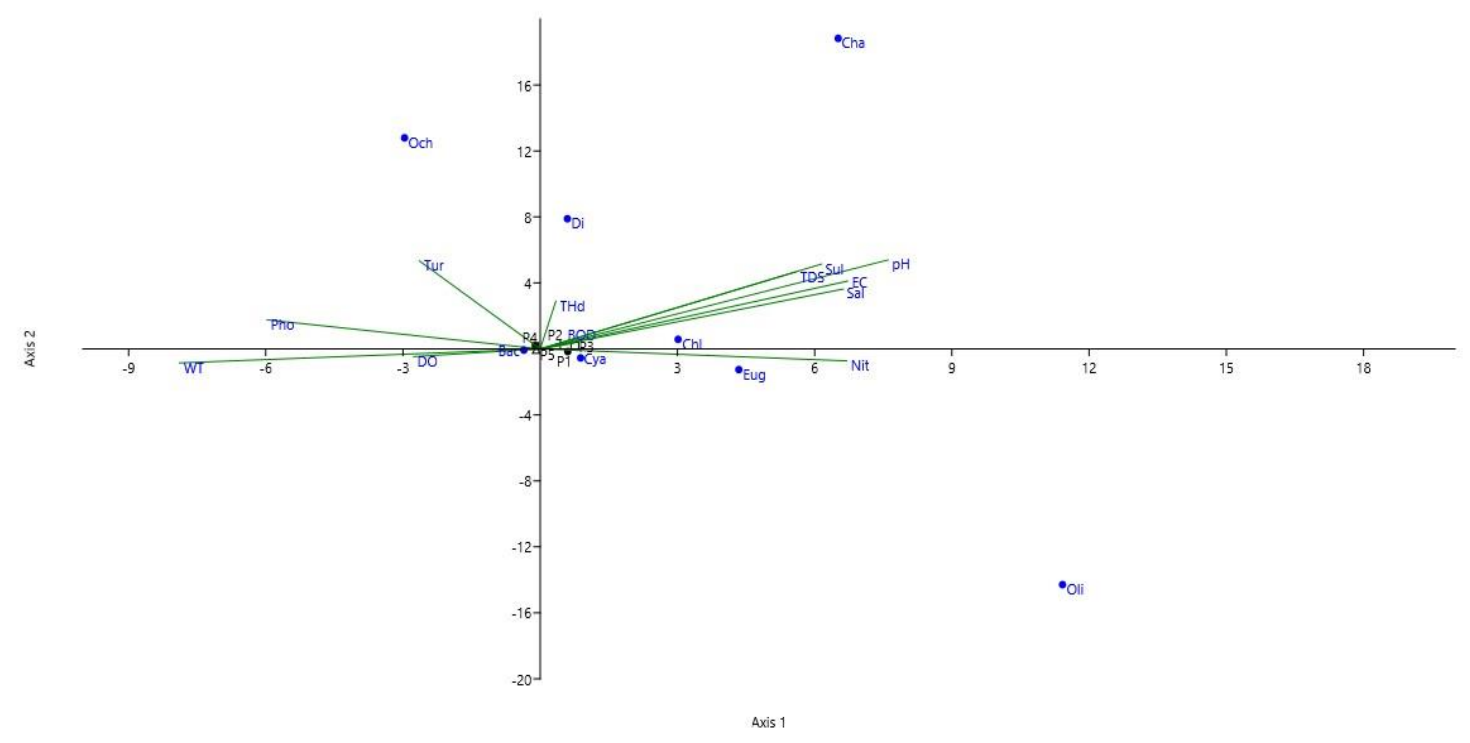

Figure 6. Canonical corresponded analysis (CCA) for phytoplankton community

However, for the zooplankton taxa (Fig. 7) there was a strong negative correlation between cyclopoida taxa and water temperature at axis 1 . Also at axis 1 , positive 
association between biological oxygen demand (BOD), dissolve oxygen (DO) and rotifers was observed. Importantly, $\mathrm{pH}, \mathrm{EC}$, salinity, turbidity, sulphate and nitrate showed strong negative association with harpacticoida taxa during the study.

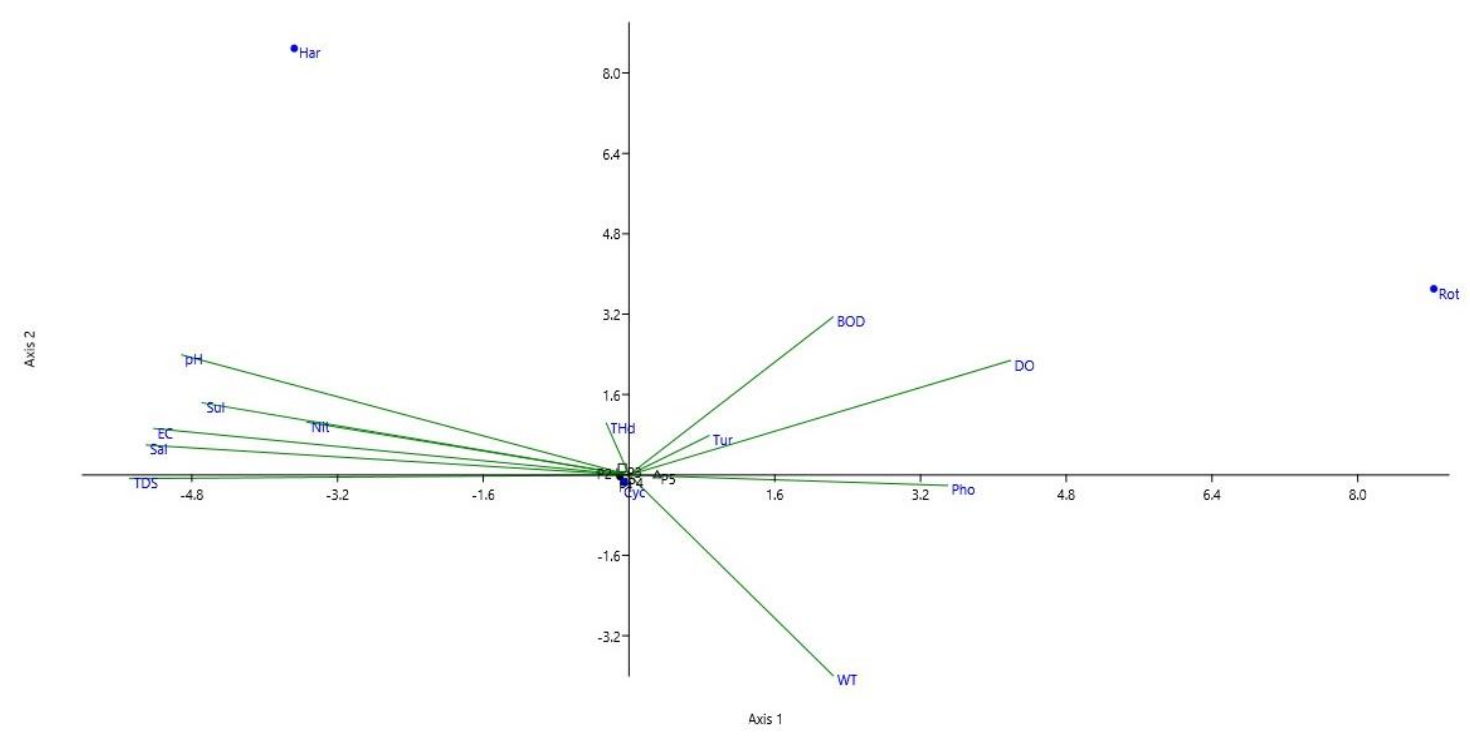

Figure 7. Canonical corresponded analysis (CCA) for zooplankton community

\section{Discussion}

The result of phytoplankton showed high species diversity, abundance and a robust community structure. In a given healthy aquatic environment, phytoplankton high population density is expected particularly in the shallow water depth rich in nutrient materials (Castro and Huber, 1997; Kadiri, 2006; Davies, 2009; Cloern et al., 2014; Dalu et al., 2016) except otherwise in contaminated or polluted waters. The dominance of Bacillariophyta division amongst the eight divisions recorded in this study corroborate the study by Kadiri (2006) and Egborge et al. (2001) in the western Niger Delta explaining that most inter-tidal waters in the zone are likely to be in this nature. Davies (2009) demonstrated that day light event (sun shine rays) play very important role in the production activities of both the primary and secondary producers as the latter is dependent on the former. The taxa among the division Bacillariophyta which were well represented across the study irrespective of the prevailing season were; Bacillaria paradoxa, Flagillaria sp and Synedra acus and were occasionally sampled at stations 1, 2, 3 and 5 respectively. The reason why the divisions Dinophyta, Ochrophyta and Oligohymenophorea recorded one species each throughout this study could not be well ascertained. However, the pattern of phytoplankton diversity is similar to what was reported (Kadiri, 2006) across waterways in the western Niger Delta of Nigeria. The pattern of diversity and species abundance observed in this study whereby some stations did not record any individual was affirmed by the output of the performance of diversity indices such as species richness and Shannon wiener diversity, and was earlier acclaimed (Kadiri, 2006 in the Niger Delta; and Lancelot and Muylaert, 2011). Environmental conditions such as temperature, $\mathrm{pH}, \mathrm{DO}, \mathrm{BOD}$, salinity and EC in the surface water of Asarama estuary greatly influenced the production (particularly of phytoplankton), distribution and stability of the biotic community (typical for the zooplankton). 
The zooplankton of Asarama estuary were in conformity to what have been documented for some coastal or brackish waters in the Niger Delta of Nigeria (Davies, 2009; Ogbuagu et al., 2011). Though, the zooplankton community structure had more taxa of the sub-class copepod which consist of the order cyclopoida and harpaticoida, and the class rotifer respectively. The dominance of copepods in this study as a finding is in consistency with some earlier studies (Kolo et al., 2001; Davies, 2009) in Nigerian coastal environments and Hwang et al. (2010) for a study in Taiwan. Zooplanktoncyclopoida was represented by seven species of which; Thermocyclops neglectus and Mesocyclops bodanicola were the most dominant and abundant species recorded across the five sampled stations. Shizopera neglecta (Harpacticoida) and the two species of Rotifers only (Keratella hiemalis and Rotaria neptunia) were recorded at station 4. We strongly attribute this phenomena to the presence of soot and oil film on the surface water which could perhaps inhibit the activities of primary producers. The restriction of the occurrence of Rotifers species to stations 3 and 5 only could not be fully ascertained in this study. Importantly, the dominance of copepods in a given aquatic environment as in this study, is an indication of a perturbed or stressed environment (Davies, 2009). Imoobe and Adeyinka (2009) reported a high species diversity for rotifers and copepods for an inland stream in Benin, Nigeria, which proved contrary to our findings for the same group of zooplankton. Environmental perturbation was found to be associated with Asarama estuary resulting from intense human activities such as transportation/navigation and over harvesting of periwinkle ( $T$. fuscatus). Despite the diversity of phytoplankton, the zooplankton diversity was low however, a situation that was least expected since Asarama estuary is typically a shallow inter-tidal ecosystem rich in nutrient resulting in high productivity in the littoral zone. However, we believe that the effect of vertical migration in zooplankton and the choice of sampling the subsurface water using qualitative method only could have accounted for the low zooplankton species abundance and species composition.

The application of canonical correspondence analysis in data analysis (Fig. 7) has been used as clarification method to establish any relationships or associations between biota and environmental variables in recent ecological studies (Lengendr and Lengendr, 1998; Yang et al., 2014 and Uwagbae et al., 2017). The positive association between BOD, DO and members of the rotifer group suggests that they have affinity for environment rich in dissolved oxygen as well as requiring low biological oxygen demand for decomposition activity. This finding was not farfetched from the observation in the Asarama estuary as there were a lot of decomposing woods and leaf litter, and dead mollusc shells in the river bed. The positive relationship that was observed between DO, BOD and rotifers was truly reflected at station 5 of Tables 1 and 2 in which case, DO and BOD had higher mean concentrations. In addition, environmental perturbation was lesser at station 5 except for dead nipa palm trees remains. Meanwhile, the negative association or correlation between harpacticoida species with several environmental factors such as salinity, EC, nutrient and $\mathrm{pH}$; foretell that they may be more tolerant to waters of very low salinity and electrical conductivity with moderate nutrient such as in inland waters. Conversely, it also suggested that total hydrocarbon content affected abundance of the already mentioned taxa. This is evident and further explains the phenomenon of the low species abundance at station 4 in particular. The same negative correlated relationship was observed for $\mathrm{pH}$ and rotifers, implying that they perhaps prefer habitats with non-acidic $\mathrm{pH}$ condition (i.e. between 6.8 and 8.2) which is contrary to our finding. Equally, at station 4, CCA proved that 
cyclopoida-zooplankton were negatively influenced by water temperature, which is attributed to the total effect of direct sun heating of the littoral zone and the due to the absence of floral community that plays temperature regulation role by providing canopy effect as well as due to the presence of soot and petroleum slick on the water surface. The combination of these environmental factors can greatly influence the abundance, diversity and community structure of biota in any give aquatic ecosystem. The work by Lancelot and Muylaert (2011) satisfactorily explains the relationships between light, temperature and turbidity in shallow estuaries which supports our finding on the negative correlation between zooplankton and water temperature in the study area. However, it could also be attributed to the phenomena of tidal action still in shallow estuaries.

\section{Conclusion}

We conclude that the species diversity and composition for zooplankton was very low when compared with the result of the phytoplankton diversity in this study. The low zooplankton composition and diversity may be attributed to the phenomenon of vertical migration in shallow water coupled with the choice of sampling which was strictly restricted to the subsurface water considering the effect of sun rays on the shallow water in terms of increasing productivity.

However, the abundance of phytoplankton and diversity is attributed to the high effect of sun light and nutrient enrichment of the shallow estuary. Nevertheless, the indiscriminate human activities such as the unregulated transportation of goods and services, shipment of petroleum fractions from an illegal local refinery at upstream (though, they have relocated their activities as at early 2017) via wooden boats including bivalve mariculture, tend to pose danger to the biodiversity sustainability, and the environmental quality. Though this study is preliminary, we advocate that a major funded survey on the biodiversity of Asarama estuary should be carried out particularly on the influence of environmental factors on the fauna and flora organisms with a broadened scope. Besides, a comprehensive study on the physicochemical oceanography of Asarama estuary is of paramount importance amidst the rapid developments in the Niger Delta of Nigeria.

It is also very important to have a formal environmental legislature in place that seeks to protect the estuary and its kinds in the region, which allows its sustainable use of the resources amongst the locals. This way, the indiscriminate human impact on the environment would be greatly reduced sustainably.

Acknowledgements. We (particularly ARD and MAU) are very thankful to Duke University and Oak Foundation of the United States for the award of Duke University Marine Laboratory mini grant for marine conservation project, which greatly supported this work. We are grateful to the following persons: Obediah Owoh (Asarama community liaison officer and local curator), Godswill Amos (boat coxswain), Ikechuckwu Odoemenam (local diver) and Hakeem Okunola (taxi driver) for their various assistance during this project. We equally extend our thanks to Mr Isaac Adewole, a research fellow of the department of Animal and Environmental Biology, University of Benin, Benin for assisting in the laboratory work. 


\section{REFERENCES}

[1] Ansa, E. J., Francis, A. (2007): Sediment characteristics of the Andoni Flats, Niger delta, Nigeria. - Journal of Applied Science and Environmental Management 11(3): 21-25.

[2] Castro, P., Huber, M. E. (1997): Marine Biology. 2nd Ed. - The WCB/McGraw-Hill Company, New York.

[3] Carlsson, P. E., Tester, G. P., Boni, L. (1995): Influences of riverine humic substances on bacteria, protozoa, phytoplankton, and copepods in a coastal plankton community. Marine Ecology Progress Series 127: 213-221.

[4] Cearreta, A., Irabien, M. J., Leorri, E., Yusta, I., Croudace, I. W., Cundy, A. B. (2000). Recent anthropogenic impacts on the Bilbao Estuary, northern Spain: geochemical and microfaunal evidence. - Estuary Coastal and Shelf Science 50: 571-592.

[5] Cloern, J. E, Foster, S. Q., Kleckner, A. E. (2014): Phytoplankton primary production in the World's estuarine-coastal ecosystems. - Biogeosciences 11: 2477-2501.

[6] Conde, D., Bonilla, S., Aubriot, L., de León, R., Pintos, W. (2007): Relative contribution of planktonic and benthic microalgae production in a eutrophic coastal lagoon of South America. - J. Limnol. 78: 202-212.

[7] Dalal, S. G., Goswami, S. C. (2001): Temporal and ephemeral variations in copepod Community in the estuaries of Mandovi and Zuari - west coast of India. - Journal of Plankton Research 23: 19-26.

[8] Dalu, T., Bere, T., Froneman, P. W. (2016): Assessment of water quality based on diatom indices in a small temperate river system, Kowie River, South Africa. - Water SA 42(2): 183-193.

[9] Davies, O. A. (2009): Spatio-temporal distribution, abundance and species composition of zooplankton of Woji-Okpoka creek, Port Harcourt, Nigeria. - Research Journal of Applied Sciences, Engineering and Technology 1(2): 14-34.

[10] Davies, O. A., Abowei, J. F. N., Tawari, C. C. (2009): Phytoplankton community of Elechi creek, Niger delta, Nigeria. A nutrient-polluted tropical creek. - American Journal of Applied Sciences 6(6): 1143-1152.

[11] Eaton, A. D., Clesceri, L. S., Rice, E. W., Greenberg, A. E., Franson, M. A. H. (2005): Standard Methods for the Examination of Water and Wastewater. 21st Ed. - APHA, Washington, DC.

[12] Egborge, A. B. M., Idu, M. C., Kadiri, M. (2001): The Plants. - In Egborge, A.B.M. (Ed): Water Pollution in Nigeria. Vol. 2. Pollution of Warri River at Opete. Ben Miller Books Ltd, Nigeria, pp. 64-75.

[13] Elliott, M., De Jonge, V. N. (2002): The management of nutrients and potential Eutrophication in Estuaries and other restricted water bodies. - Hydrobiologia 475/476: 513-524.

[14] Elliott, M., McLusky, D. S. (2002): The need for definition in understanding estuaries. Estuary Coastal and Shelf Science 55: 815-827.

[15] Froneman, P. W. (2004): Zooplankton community structure and biomass in a South African temporarily open/closed estuary. - Estuary Coastal and Shelf Science 60: 125132.

[16] Godhantaraman, N., Uye, S. (2003): Geographical and seasonal variations in taxonomic composition, abundance and biomass of microzooplankton across a brackish-water lagoonal system of Japan. - Journal of Plankton Research 25(5): 465-482.

[17] Hammer, Ø., Harper, D. A. T., Ryan, D. A. T. (2001): PAST: Palaeontological Statistics Software Package for Education and data analysis. - Palaeontologia Electronica 4: 1-9. http://clade.ansp.org/entomology/mongolia/maishome.html.

[18] Hwang, J. S., Kumar, R., Hsieh, C. W., Kuo, A., Souissi, S., Hsu, M. H., Wu, J. T., Liu, W. C., Wang, C. F., Chen, Q. C. (2010): Patterns of zooplankton distribution along the marine, estuarine, and riverine portions of the Danshuei ecosystem in northern Taiwan. Zoological Studies 49(3): 335-352. 
[19] Imoobe, T. O. T., Adeyinka, M. L. (2009): Zooplankton-based assessment of the trophic state of a tropical forest river in Nigeria. - Archive for Biological Sciences 61(4): 733740 .

[20] Jeje, C. Y., Fernando, C. H. (1986): A Practical Guide to the Identification of Nigerian Zooplankton. - Kainji Lake Research Institute Nigeria.

[21] Kadiri, M. O. (2006): Phytoplankton survey in the Western Niger Delta, Nigeria. African Journal of Environment and Pollution Health 5(1): 48-58.

[22] Kolo, R. J., Mani, I. A., Musa, H. A. (2001): Effects of different fertilizers on plankton productivity in earthen ponds. - Journal of Aquatic Science 16(2): 127-131.

[23] Lancelot, C., Muylaert, K. (2011): Trends in Estuarine Phytoplankton Ecology. - Elsevier Inc., Amsterdam.

[24] Legendre, P., Legendre, L. (1998): Developments in Environmental Modelling. Numerical Ecology 24: 41-46.

[25] Needham, J. G., Needham, P. R. (1978): A Guide to the Study of Freshwater Biological Holden. - Devy Inc., San Francisco.

[26] Newell, G. E., Newell, R. C. (1966): - Marine Plankton: A Practical Guide. Revised Edition. - Hutchinson, London.

[27] Ogbuagu, D. H., Ayoade, A. A., AC-Chukwuocha, N. B. (2011): Spatial dynamics in physicochemistry and bacterio- and myco-plankton assemblages of Imo River in a Niger Delta community in Nigeria. - African Journal of Microbiology Research 5(8): 872-887. DOI: $10.5897 /$ AJMR10.612.

[28] Opute, I. F. (1990): Phytoplankton flora in the Warri/Forcados Estuary of Southern Nigeria. - Hydrobiologia 208: 101-109.

[29] Pearl, H. W., Rossignol, K. L., Hall, N. S., Peierls, P. L., Wetz, M. S. (2010): Phytoplankton Community indicators of short and long term ecological changes in the anthropogenically and climatically impacted Neuse River estuary, North Carolina, USA. - Estuaries and Coast 33: 485-497.

[30] Prescott, G. W. (1975): How to Know the Freshwater Algae. - Brown Co. Publishers, Dubuque, Iowa.

[31] Townsend, C. R., Harper, J. L., Begon, M. (2000): Essentials of Ecology. 3rd Edn. Blackwell Science Publishers, London.

[32] Tan, Y., Huang, L., Chen, Q. C., Huang, X. (2004): Seasonal variation in zooplankton composition and grazing impact on phytoplankton standing stock in the Pearl River Estuary, China. - Continental Shelf Research 24: 1949-1968.

[33] Thor, P., Nielson, T. G., Tiselius, P., Juul-Pederson, T., Michel, C., Møller, E. F. et al. (2005): Post-spring bloom community structure of pelagic copepods in the Disko Bay, Western Greenland. - Journal of Plankton Research 27: 341-356.

[34] Uwagbae, M. A., El Surtasi, E. I., Rotimi, J., Dirisu, A. R., Gbarakoro, T. N., Richard, G., Oratokhai, R. A., Agwu, J. E. (2017): Land Reclamation, Soil Quality and Agriculture. In: Bharti, P. K., Chauhan, A. (eds.) Upshots of Age of Oil Spill and Influence of Physico-Chemical Properties on the Diversities of Soil Dwelling Insects. Discovery Publishing House, Pvt, Ltd, New Delhi, pp. 22-45.

[35] Van De Velde, I. (1984): Revision of the African species of the genus - Mesocyclops Sars, 1914 (Copepoda; Cyclopidae). - Hydrobiologia 109: 3-66.

[36] Waniek, J. J. (2003): The role of physical forcing in initiation of spring blooms in the northeast Atlantic. - Journal of Marine Systems 39: 57-82.

[37] Waniek, J. J., Holliday, N. P., Davidson, R., Brown, L., Henson, S. A. (2005): Freshwater control of onset and species composition of Greenland shelf spring bloom. - Marine Ecology Progress Series 288: 45-57.

[38] Yang, Z. F., Sun, T., Zhao, R. (2014): Environmental flow assessments in estuaries related to preference of phytoplankton. - Hydrology and Earth System Sciences 18: 1785-1791. 